\title{
ON DOUBLY WARPED PRODUCTS
}

\author{
Sibel Gerdan AYDIN and Hakan Mete TAŞTAN
}

İstanbul University, Department of Mathematics, 34134, Vezneciler, İstanbul-TURKEY.

\begin{abstract}
We give a new characterization for doubly warped products by using the geometry of their canonical foliations intersecting perpendicularly. We also give a necessary and sufficient condition for a doubly warped product to be a warped or a direct product. As a result, we prove the non-existence of Einstein proper doubly warped product pseudo-Riemannian manifold of dimension grater or equal than 4.
\end{abstract}

The characters in abstract should be between 500 to 5000

\section{Introduction}

The notion of warped product of pseudo-Riemannian manifolds was defined by Bishop and O' Neill in [2] in order to construct a large class of complete manifolds of negative curvature. In fact, this notion appeared in the literature before 2 under the name of semi-reducible spaces [10. Also, this notion is a natural and fruitful generalization of the notion of direct (or Riemannian) product. One of the reasons why warped products have been studied actively is that they play very important roles in physics as well as in differential geometry, especially in the theory of relativity. In fact, the standard space-time models such as Robertson-Walker, Schwarschild, static and Kruskal are warped products. Moreover, the simplest models of neighborhoods of stars and black holes are warped products [12.

In this paper, we first prove a existence theorem for doubly warped products. Secondly, we give a necessary and sufficient condition, called the mixed Ricci-flatness for a doubly warped product to be a warped or a direct product. In order to achieved this, we use a result of [1] or 14] concerning Ricci tensor of a doubly warped product. Then by using this result, we prove the non-existence of Einstein

2020 Mathematics Subject Classification. 53C05, 53C25, 53C50.

Keywords and phrases. Doubly warped product, warped product, direct product, Einstein manifold.

\sibel.gerdan@istanbul.edu.tr-Corresponding author; hakmete@istanbul.edu.tr

(D) 0000-0001-5278-6066; 0000-0002-0773-9305.

(C)2020 Ankara University Communications Faculty of Sciences University of Ankara-Series A1 Mathematics and Statistics 
doubly warped product pseudo-Riemannian manifold of dimension $\geq 4$ in proper case.

\section{Preliminaries}

Let $M_{1}$ and $M_{2}$ be any pseudo-Riemannian manifolds endowed with pseudoRiemannian metric tensors $g_{1}$ and $g_{2}$ respectively, and let $f_{1}$ and $f_{2}$ are positive smooth functions defined on $M_{1} \times M_{2}$. Also $\pi_{1}$ and $\pi_{2}$ are canonical projections of $M_{1} \times M_{2}$ onto $M_{1}$ and $M_{2}$, respectively. Then the doubly twisted product [13 $f_{2} M_{1} \times f_{1} M_{2}$ of $\left(M_{1}, g_{1}\right)$ and $\left(M_{2}, g_{2}\right)$ is the product manifold $M=M_{1} \times \bar{M}_{2}$ equipped with metric $g=f_{2}^{2} g_{1} \oplus f_{1}^{2} g_{2}$ given by

$$
g=f_{2}^{2} \pi_{1}^{*}\left(g_{1}\right)+f_{1}^{2} \pi_{2}^{*}\left(g_{2}\right)
$$

where $\pi_{i}^{*}\left(g_{i}\right)$ is the pullback of $g_{i}$ via $\pi_{i}$ for $i=1,2$. Each function $f_{i}$ is called a twisting function of the doubly twisted product $\left(f_{2} M_{1} \times{ }_{f_{1}} M_{2}, g\right)$. In this case, if either $f_{1} \equiv 1$ or $f_{2} \equiv 1$, but not both, then we obtain a twisted product 4 .

If the twisting functions $f_{1}$ and $f_{2}$ only depend on the points of $M_{1}$ and $M_{2}$ respectively, then $\left(f_{2} M_{1} \times_{f_{1}} M_{2}, g\right)$ is called a doubly warped product pseudo-Riemannian manifold [6]. The functions $f_{1}$ and $f_{2}$ are called warping functions of doubly warped product. In which case, if either $f_{1} \equiv 1$ or $f_{2} \equiv 1$, but not both, then we obtain a warped product [2]. If both $f_{1} \equiv 1$ and $f_{2} \equiv 1$, then we get a direct (or Riemannian) product 5. If neither $f_{1}$ nor $f_{2}$ is constant, then we say that $\left(f_{2} M_{1} \times f_{f_{1}} M_{2}, g\right)$ is proper doubly warped product pseudo-Riemannian manifold.

Let $\left(f_{2} M_{1} \times{ }_{f_{1}} M_{2}, g\right)$ be a doubly warped product manifold with the Levi-Civita connection $\nabla$ and $\nabla^{i}$ denote the Levi-Civita connection of $M_{i}$ for $i \in\{1,2\}$. By usual convenience, we denote the set of lifts of vector fields on $M_{i}$ by $\mathfrak{L}\left(M_{i}\right)$ and use the same notation for a vector field and for its lift. On the other hand, each $\pi_{i}$ is a (positive) homothety, so it preserves the Levi-Civita connection. Thus, there is no confusion using the same notation for a connection on $M_{i}$ and for its pullback via $\pi_{i}$. Then, the covariant derivative formulas for a doubly warped product manifold 6 are given as:

$$
\begin{gathered}
\nabla_{X} Y=\nabla_{X}^{1} Y-g(X, Y) \nabla\left(\ln \left(f_{2} \circ \pi_{2}\right)\right), \\
\nabla_{X} V=\nabla_{V} X=V\left(\ln \left(f_{2} \circ \pi_{2}\right)\right) X+X\left(\ln \left(f_{1} \circ \pi_{1}\right)\right) V, \\
\nabla_{U} V=\nabla_{U}^{2} V-g(U, V) \nabla\left(\ln \left(f_{1} \circ \pi_{1}\right)\right)
\end{gathered}
$$

for $X, Y \in \mathfrak{L}\left(M_{1}\right)$ and $U, V \in \mathfrak{L}\left(M_{2}\right)$. Moreover, $M_{1} \times\left\{p_{2}\right\}$ and $\left\{p_{1}\right\} \times M_{2}$ are totally umbilical submanifolds with closed mean curvature vector fields in $\left(f_{2} M_{1} \times_{f_{1}} M_{2}, g\right)$ [1], where $p_{1} \in M_{1}$ and $p_{2} \in M_{2}$.

Remark 1. From now on, we will use the same symbols for warping functions and their pullbacks. 
Next, we recall that some facts for later use.

Let $M$ a pseudo-Riemannian manifold with metric tensor $g$. The Ricci tensor of $M$ is a symmetric $(0,2)$ type tensor defined by

$$
\operatorname{Ric}(X, Y)=\sum_{i=1}^{m} g\left(E_{i}, E_{i}\right) g\left(R\left(E_{i}, X\right) Y, E_{i}\right),
$$

where $X$ and $Y$ are smooth vector fields on $M,\left\{E_{1}, \ldots, E_{m}\right\}$ is an orthonormal frame field on the set of all smooth vector fields on $M$ and $R$ is Riemann curvature tensor of $M$ defined by

$$
R(X, Y) Z=\nabla_{X} \nabla_{Y} Z-\nabla_{Y} \nabla_{X} Z-\nabla_{[X, Y]} Z,
$$

here $\nabla$ is the Levi-Civita connection with respect to the metric $g$. For more details, see [5].

For the Ricci tensor of a doubly warped product $f_{2} M_{1} \times{ }_{f_{1}} M_{2}$ with $\operatorname{dim}\left(M_{1}\right)=m_{1}>1$ and $\operatorname{dim}\left(M_{2}\right)=m_{2}>1$, we have the following result from Theorem 2.5.2 of [14] or the equation (2.19) of [1],

$$
\operatorname{Ric}(X, V)=\left(m_{1}+m_{2}-2\right)\left(\frac{X f_{1}}{f_{1}}\right)\left(\frac{V f_{2}}{f_{2}}\right),
$$

where $X \in \mathcal{L}\left(M_{1}\right)$ and $V \in \mathcal{L}\left(M_{2}\right)$.

\section{Main Results}

We need the following two facts to prove the first main theorem.

Lemma 2. (Proposition 3-a [13]) Let $M=M_{1} \times M_{2}$ and call $\left(\mathcal{D}_{1}, \mathcal{D}_{2}\right)$ the canonical foliations. Suppose that $g$ is a pseudo-Riemann metric such that $\mathcal{D}_{1}$ and $\mathcal{D}_{2}$ are orthogonal. Then $(M, g)$ is a doubly twisted product ${ }_{f_{2}} M_{1} \times_{f_{2}} M_{2}$ if and only if $\mathcal{D}_{1}$ and $\mathcal{D}_{2}$ are totally umbilic.

Lemma 3. (Lemma 2.3 [9]) Let $f_{2} M_{1} \times_{f_{2}} M_{2}$ be a doubly twisted product. It is a doubly warped product if and only if the mean curvature vector fields of canonical foliations are closed.

We are ready to prove the main theorem.

Theorem 4. Let $(M, g)$ be a pseudo-Riemannian manifold and $\mathcal{D}_{1}$ and $\mathcal{D}_{2}$ be canonical foliations on $M$. Suppose that $\mathcal{D}_{1}$ and $\mathcal{D}_{2}$ intersect perpendicularly everywhere. Then $g$ is the metric of doubly warped product ${ }_{f_{2}} M_{1} \times_{f_{1}} M_{2}$ if and only if there exists a smooth function $\mu_{1}$ (resp. $\mu_{2}$ ) on $M_{1}$ (resp. $M_{2}$ ) such that for any $Z \in \mathcal{L}\left(M_{1}\right)$ and $W \in \mathcal{L}\left(M_{2}\right)$, we have

$$
\mathcal{L}_{W} g=2 W\left[\mu_{2}\right] g \quad \text { on } \quad M_{1}
$$


and

$$
\mathcal{L}_{Z} g=2 Z\left[\mu_{1}\right] g \text { on } M_{2},
$$

where $\mathcal{L}_{W}$ is the Lie derivative with respect to $W$ and $M_{1}$ (resp. $M_{2}$ ) is the integral manifold of $\mathcal{D}_{1}$ (resp. $\mathcal{D}_{2}$ ).

Proof. Let ${ }_{f_{2}} M_{1} \times{ }_{f_{1}} M_{2}$ be a doubly warped product with the metric $g=f_{2}^{2} g_{1} \oplus$ $f_{1}^{2} g_{2}$. Then using the Lie derivative formula, for any $X, Y, Z \in \mathfrak{L}\left(M_{1}\right)$ and $U, V, W \in$ $\mathfrak{L}\left(M_{2}\right)$, we have

$$
\left(\mathcal{L}_{W} g\right)(X, Y)=-2 g\left(h_{1}(X, Y), W\right)
$$

and

$$
\left(\mathcal{L}_{Z} g\right)(U, V)=-2 g\left(h_{2}(U, V), Z\right)
$$

where $h_{1}$ (resp. $h_{2}$ ) denotes the second fundamental form of $M_{1}$ (resp. $M_{2}$ ), (e.g. see $[3$, p. 195]). By using (1) and (3), we obtain

$$
\left(\mathcal{L}_{W} g\right)(X, Y)=-2 g\left(-g(X, Y) \nabla\left(\ln f_{2}\right), W\right)
$$

and

$$
\left(\mathcal{L}_{Z} g\right)(U, V)=-2 g\left(-g(U, V) \nabla\left(\ln f_{1}\right), Z\right)
$$

from (9) and (10), respectively. By direct calculation, we get

$$
\left(\mathcal{L}_{W} g\right)(X, Y)=2 W\left[\ln f_{2}\right] g(X, Y)
$$

and

$$
\left(\mathcal{L}_{Z} g\right)(U, V)=2 Z\left[\ln f_{1}\right] g(U, V)
$$

from (11) and (12), respectively. Thus, we find the assertion (7) for $\mu_{2}=\ln f_{2}$ from (13) and the assertion (8) for $\mu_{1}=\ln f_{1}$ from (14).

Conversely, suppose that the conditions $(7)$ and $(8)$ hold. Then for any $X, Y, Z \in$ $\mathfrak{L}\left(M_{1}\right)$ and $U, V, W \in \mathfrak{L}\left(M_{2}\right)$, using (7) (10), we have

$$
-2 g\left(h_{1}(X, Y), W\right)=2 W\left[\mu_{2}\right] g(X, Y)
$$

and

$$
-2 g\left(h_{2}(U, V), Z\right)=2 Z\left[\mu_{1}\right] g(U, V) .
$$

After some calculation, we obtain

$$
g\left(h_{1}(X, Y), W\right)=g\left(-g(X, Y) \nabla \mu_{2}, W\right)
$$

and

$$
g\left(h_{2}(U, V), Z\right)=g\left(-g(U, V) \nabla \mu_{1}, Z\right)
$$

from 15 and (16), respectively. We get

$$
h_{1}(X, Y)=-g(X, Y) \nabla \mu_{2}
$$

and

$$
h_{2}(U, V)=-g(U, V) \nabla \mu_{1}
$$

from (17) and 18), respectively. The equation (19) (resp. (20p) tells us the canonical foliation $\mathcal{D}_{1}$ (resp. $\left.\mathcal{D}_{2}\right)$ is totally umbilical with the mean curvature vector 
field $-\nabla \mu_{2}$ (resp. $-\nabla \mu_{1}$ ). Moreover, the mean curvature vector field $-\nabla \mu_{1}$ (resp. $\left.-\nabla \mu_{2}\right)$ is closed, since its dual 1 -form $-d \mu_{1}$ (resp. $\left.-d \mu_{2}\right)$ is closed. Thus by Lemmas 2 and $3, g$ is the metric of a doubly warped product $f_{2} M_{1} \times_{f_{1}} M_{2}$.

Before going to give the second main result, let recall the definition of mixed Ricci-flatness.

Let $M={ }_{f_{2}} M_{1} \times{ }_{f_{1}} M_{2}$ be a doubly warped product pseudo-Riemannian manifold with metric tensor $g=f_{2}^{2} g_{1} \oplus f_{1}^{2} g_{2}$. Then we say that $(M, g)$ is mixed Ricci-flat, if we have $\operatorname{Ric}(X, V)=0$ for every $X \in \mathcal{L}\left(M_{1}\right)$ and $V \in \mathcal{L}\left(M_{2}\right)$ ].

Theorem 5. Let $f_{2} M_{1} \times_{f_{1}} M_{2}$ be a doubly warped product of $\left(M_{1}, g_{1}\right)$ and $\left(M_{2}, g_{2}\right)$ with warping functions $f_{1}$ and $f_{2}$ and $\operatorname{dim}\left(M_{1}\right)=m_{1}>1$ and $\operatorname{dim}\left(M_{2}\right)=m_{2}>1$. Then ${ }_{f_{2}} M_{1} \times_{f_{1}} M_{2}$ is mixed Ricci-flat if and only if

(1) either $f_{2} M_{1} \times{ }_{f_{1}} M_{2}$ can be expressed as a warped product ${ }_{f_{2}} M_{1} \times M_{2}$ of $\left(M_{1}, \tilde{g_{1}}\right)$ and $\left(M_{2}, g_{2}\right)$ with warping function $f_{2}$, where $\tilde{g_{2}}=k_{1}^{2} g_{2}$ for some positive constant $k_{1}$, or

(2) either $f_{2} M_{1} \times_{f_{1}} M_{2}$ can be expressed as a warped product $M_{1} \times_{f_{1}} M_{2}$ of $\left(M_{1}, g_{1}\right)$ and $\left(M_{2}, \hat{g_{2}}\right)$ with warping function $f_{1}$, where $\hat{g_{1}}=k_{2}^{2} g_{1}$ for some positive constant $k_{2}$, or

(3) $f_{2} M_{1} \times_{f_{1}} M_{2}$ is a direct product $M_{1} \times M_{2}$ of $\left(M_{1}, \overline{g_{1}}\right)$ and $\left(M_{2}, \overline{g_{2}}\right)$, where $\overline{g_{1}}=c_{2}^{2} g_{1}$ and $\overline{g_{2}}=c_{1}^{2} g_{2}$ for some positive constants $c_{1}$ and $c_{2}$.

Proof. If ${ }_{f_{2}} M_{1} \times_{f_{1}} M_{2}$ is mixed Ricci-flat, then we have $\operatorname{Ric}(X, V)=0$ for all $X \in \mathcal{L}\left(M_{1}\right)$ and $V \in \mathcal{L}\left(M_{2}\right)$. Thus, by the hypothesis and (6), we obtain

$$
\left(X f_{1}\right)\left(V f_{2}\right)=0
$$

for all $X \in \mathcal{L}\left(M_{1}\right)$ and $V \in \mathcal{L}\left(M_{2}\right)$. There are three different cases.

Case 1. $X f_{1}=0$ and $V f_{2} \neq 0$.

Hence, we find $f_{1}=k_{1}$ for some positive constant $k_{1}$. Thus, we can write $g=f_{2}^{2} g_{1} \oplus \tilde{g_{2}}$, where $\tilde{g_{2}}=k_{1}^{2} g_{2}$, that is $f_{2} M_{1} \times_{f_{1}} M_{2}$ can be expressed as a warped product $f_{2} M_{1} \times M_{2}$ with warping function $f_{2}$, where the metric tensor of $M_{2}$ is $\tilde{g_{2}}$ given above. This is (1).

Case 2. $X f_{1} \neq 0$ and $V f_{2}=0$.

Similarly, $f_{2} M_{1} \times_{f_{1}} M_{2}$ can be expressed as a warped product $M_{1} \times_{f_{1}} M_{2}$ with warping function $f_{1}$, where the metric tensor of this warped product $M_{1} \times f_{1} M_{2}$ $M_{2}$ is $g=\hat{g_{1}} \oplus f_{1}^{2} g_{2}$ such that $\hat{g_{1}}=k_{2}^{2} g_{1}$ for some positive constant $k_{2}$, so we get (2). 
Case 3. $X f_{1}=V f_{2}=0$.

Then, it follows immediately that $f_{1}=c_{1}$ and $f_{2}=c_{2}$, where $c_{1}$ and $c_{2}$ are positive constants. Thus, it is easy to see that $f_{2} M_{1} \times_{f_{1}} M_{2}$ is a direct product $M_{1} \times M_{2}$ of $\left(M_{1}, \overline{g_{1}}\right)$ and $\left(M_{2}, \overline{g_{2}}\right)$, here $\overline{g_{1}}=c_{2}^{2} g_{1}$ and $\overline{g_{2}}=c_{1}^{2} g_{2}$. Which is (3). The converse is obvious from the equation (6).

A pseudo-Riemannian manifold $(M, g)$ is called an Einstein manifold if its Ricci tensor proportional to its metric, i.e., Ric $=\lambda g$ for some constant $\lambda$ 5. Since, the Einstein conditions leads to mixed Ricci-flatness, by our main result Theorem 5 , we have following result.

Corollary 6. There exist no Einstein proper doubly warped product pseudo-Riemannian manifold of dimension greater or equal than 4 .

Remark 7. This result was also obtained without dimension restriction in [1] by different manner, see Proposition 3.1 of [1].

Remark 8. In [8], the author asserts that the existence of Einstein doubly warped product pseudo-Riemannian manifolds, see Remark 3.3 of [8]. But Corollary 6 contradicts that result.

Remark 9. The mixed Ricci-flatness condition was also used for a twisted product to be a warped product by M. Fernández López et al [7].

Remark 10. As can be easily seen from the Preliminaries section, there exist no inclusion relation between the classes of proper twisted products and the classes of proper doubly warped products.

Remark 11. Some space-time models such as Robertson-Walker and Kruskal have the mixed Ricci-flatness property. Thus, in view of Theorem 5, these space-times cannot be further generalized to the proper doubly warped products.

Acknowledgment. This work is supported by 1001-Scientific and Technological Research Projects Funding Program of The Scientific and Technological Research Council of Turkey (TUBITAK) project number 119F179.

\section{REFERENCES}

[1] Agaoka, Y., Kim, I-B., Yeom, D.J., On doubly warped product manifolds, Mem. Fac. Integrated Arts and Sci., Hiroshimo Univ., ser. IV., 24 (1998), 1-10.

[2] Bishop, R.L., O’Neill, B., Manifolds of negative curvature, Trans. Amer. Mat. Soc., 145(1) (1969), 1-49.

[3] Blumenthal, R.A., Hebda, J.J., An analogue of the holonomy bundle for a foliated manifold, Tôhoku Math. J., 40(2) (1988), 189-197.

[4] Chen, B.Y., Geometry of submanifolds and its applicaitons, Science University of Tokyo, Tokyo, 1981.

[5] Chen, B.Y., Differential geometry of warped product manifolds and submanifolds, World Scientific, 2017. 
[6] Ehrlich, P. E., Metric deformations of Ricci and sectional curvature on compact Riemannian manifolds, Ph.D. dissertation, SUNY, Stony Brook, N.Y., 1974.

[7] Fernández López, M., García Río, E., Küpeli, D.N., Ünal, B., A curvature condition for a twisted product to be a warped product, Manuscripta Math., 106 (2001), 213-217.

[8] Gupta, P., On compact Einstein doubly warped product manifolds, Tamkang J. Math., 49(4) (2018), 267-275. doi:10.5556/j.tkjm.49.2018.2605.

[9] Gutierrez, M., Olea, B., Semi-Riemannian manifolds with a doubly warped structures, Revista Mathematica Iberoamericana, 28(1) (2012), 1-24.

[10] Kruchkovich, G.I., On semi-reducible Riemannian spaces (in Russian), Dokl. Akad. Nauk SSSR, 115 (1957), 862-865.

[11] Olea, B., Doubly warped product structures on semi-Riemannian manifolds, Ph.D. thesis, Universty of Malaga, 2009.

[12] O'Neill, B., Semi-Riemannian geometry with applications to relativity, Academic Press, San Diego, 1983.

[13] Ponge, R., Reckziegel, H., Twisted products in pseudo- Riemannian geometry, Geom. Dedicata, 48 (1993), 15-25.

[14] Ünal, B., Doubly warped products, Ph.D. thesis, Universty of Missouri-Columbia, 2000.

[15] Yano, K., Kon, M., Structures on Manifolds, World Scientific, Singapore, 1984. 